\title{
Reflection optical encoders as three-grating moiré systems
}

\author{
Daniel Crespo, Jose Alonso, and Eusebio Bernabeu
}

\begin{abstract}
Reflection optical encoders are studied as three-grating moiré systems. An analysis is made of the differences that may appear between it and the standard case in which an optical encoder is regarded as a two-grating system. (C) 2000 Optical Society of America

OCIS codes: $\quad 050.1940,050.1950,120.3940,120.4120,120.5700$.
\end{abstract}

\section{Introduction}

The most extensive types of optical encoder (linear transducers as well as rotational encoders) are based on the moiré effect.1,2 These encoders have two gratings in tandem that form a moiré fringe pattern when they are illuminated with monochromatic collimated light. When one of the gratings is displaced in the direction perpendicular to its ruling, the total light intensity that passes through the system varies periodically, and this periodic variation is used as a signal to monitor the displacement of the grating. The different parameters that affect the signal of such an encoder and determine its design and mounting tolerances have been studied thoroughly by several authors. ${ }^{3-5}$

Many of these optical encoders work in a reflection setup, ${ }^{1,6}$ as does the encoder depicted in Fig. 1. In this setup there is a transmission grating $G_{1}$ that is usually fixed to the light-emitting source and the detector and another reflection grating $G_{2}$ that is movable. The light coming from the source goes through $G_{1}$, is reflected off of $G_{2}$, and goes back again through $G_{1}$ until it impinges upon the detector. This type of reflection optical encoder actually works as a threegrating system; nevertheless, these systems typically have been studied as two-grating systems ${ }^{1}$ neglecting the effect of the light passing twice through $G_{1}$.

There have been studies of the intensity distribution that is obtained after light propagation through

The authors are with the Departamento de Optica, Universidad Complutense de Madrid, Avenida Complutense s/n, 28040 Madrid, Spain. D. Crespo's e-mail address is dcrespo@eucmos.sim.ucm.es. Received 12 January 2000; revised manuscript received 20 April 2000.

0003-6935/00/220001-09\$15.00/0

(C) 2000 Optical Society of America a two-grating system, for example, the study presented for fractional Talbot planes by Liu et al. ${ }^{7}$ or the more recent work by Wronkowski. ${ }^{8}$ We consider just the additional effect of a third grating on the observation plane to provide a complete description of the system depicted in Fig. 1.

In this paper, we study a reflection optical encoder as a three-grating system. With this approach, we study the signal of such an encoder and how it is affected by changes in the different construction parameters of the encoder. We restricted our study to only those parameters for which the presence of the third grating produces significant effects because the rest of the effects are already well known for the two-grating case..$^{8,9}$ In all these aspects, we compare our results with those obtained when the effect of the third grating is neglected, which would correspond to the case of a transmission optical encoder.

In Section 2 of this paper, we obtain expressions for the total intensity distribution that would be obtained as the output of a reflection encoder, taking into account the effect of the light passing twice through the first grating. In Section 3, we study the modulation of the signal of the encoder as a function of the distance between gratings and compare it with the two-grating case. In Section 4, we study the changes in the signal of the encoder when the light source is not aligned perfectly along the optical axis of the system and therefore the incident wave front is tilted. In Section 5, we study the changes in the signal of the encoder that appear when the light source is defocused and the incident light beam is not perfectly collimated. In Section 6 , we consider the effects of the size of the light source on the signal of the encoder and compare them with the effects in the two-grating case. Finally, in Section 7, we present experimental results from the previous calculations. 


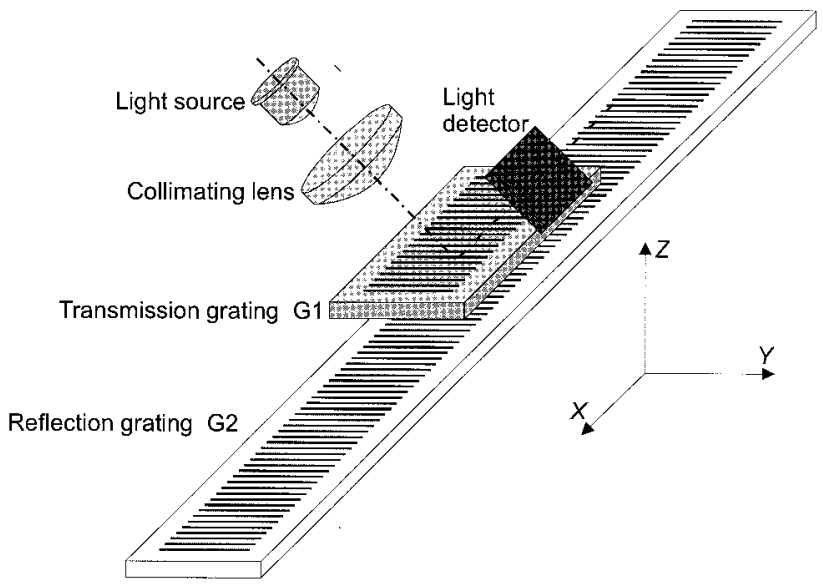

Fig. 1. Typical scheme of a moiré reflection encoder.

\section{General Analysis}

The optical system that would be equivalent to a reflection optical encoder-and that is the object of this analysis - is the one depicted in Fig. 2. $G_{1}, G_{2}$, and $G_{3}$ are Rhonchi gratings; it is assumed that they all have the same period $p$ and that they all are oriented along the $x$ axis and are parallel to each other. We call $z_{1}$ the distance between the first and the second gratings and $z_{2}$ the distance between the second and the third gratings. At the end of the calculations, we use the fact that $z_{1}=z_{2}$ in reflection encoders. This relation must be so because the first and the third gratings are, in fact, the same grating.

We have a small light source whose size, for the moment, is neglected. This light source is collimated by use of a lens with a back focal length $f$. We consider that the light source is placed on the back focal plane of the lens but that it can be shifted laterally from the optical axis by a distance $D$.

We want to study the light-intensity distribution on an observation plane located directly behind the third grating. To do this, we study the propagation through the system of a given incident light field under the Fresnel approximation. The whole problem is treated as one dimensional because we consider, as a good approximation, that the gratings have an infinite extension along the $y$ axis and that the incident light field has a constant distribution along that same axis.

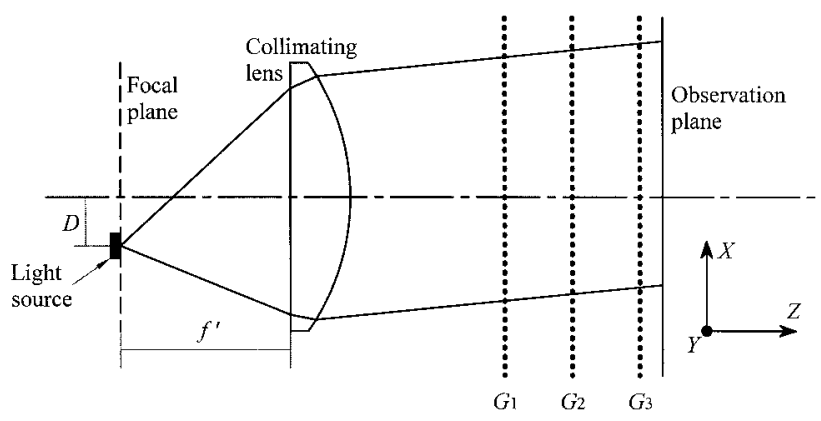

Fig. 2. Optical setup of a three-grating moiré system.
In a system like the one described above, the light incident on the system can be considered to be a monochromatic planar wave front as long as we neglect lens aberrations. This planar wave front is tilted by an angle $\theta=D / f$ with respect to the optical axis of the system. In any practical design the shift $D$ will be much smaller than $f$. Under these conditions the spatial light-field distribution directly before the plane of the first grating can be expressed as

$$
\varphi_{1 B}(x)=\exp \left(i q_{0} x\right)
$$

where $q_{0}=2 \pi \theta / \lambda$ is the spatial frequency and $\lambda$ is the wavelength of the incident light.

The effect of the first grating on the incident light can be described completely by its complex transmittance. Because the grating is periodic, its field transmittance can be written as a Fourier series. This series can be expressed as

$$
t_{1}(x)=\sum_{n} a_{n} \exp (i q n x)
$$

where $q=2 \pi / p$ is the spatial frequency of the gratings and $a_{n}$ represent the coefficients corresponding to the Fourier expansion of a Rhonchi grating. More explicitly,

$$
a_{0}=\frac{1}{2}, \quad a_{2 n+1}=\frac{(-1)^{n}}{2 n+1}, \quad a_{2 n}=0
$$

The field distribution directly after the first grating is given by

$$
\varphi_{1 A}(x)=t_{1}(x) \varphi_{1 B}(x)=\sum_{n} a_{n} \exp \left[i x\left(q n+q_{0}\right)\right]
$$

The Fourier transform of this distribution [Eq. (4)] is necessary for the following calculations. This Fourier transform (TF) can be written in the following way:

$$
\Phi_{1 A}\left(k_{x}\right)=\operatorname{TF}\left[\varphi_{1 A}(x)\right]=\sum_{n} a_{n} \delta\left(k_{x}-q n-q_{0}\right) .
$$

By use of the Fresnel propagation formula the light field propagated to the plane of the second grating is given by

$$
\begin{aligned}
\varphi_{2 B}(x)= & \int_{-\infty}^{\infty} \exp \left(i k_{x} x\right) \exp \left(-i k_{x}^{2} \frac{z_{1}}{2 k}\right) \sum_{n} a_{n} \delta\left(k_{x}-q n\right. \\
& \left.-q_{0}\right) \\
= & \sum_{n} a_{n} \exp \left[i x\left(q n+q_{0}\right)\right] \exp \left[-i\left(q n+q_{0}\right)^{2} \frac{z_{1}}{2 k}\right],
\end{aligned}
$$

where $z_{1}$ is the distance between the first and the second grating and $k=2 \pi / \lambda$. 
The second grating is also characterized by its complex transmittance. In this case it is given by

$$
t_{2}(x)=\sum_{m} a_{m} \exp (i \phi m) \exp (i q m x),
$$

where the term $\exp (i \phi m)$ is introduced to consider the possible displacement of the second grating along the $x$ axis. A displacement $\delta x$ along the positive direction of this axis will correspond to a value of $\phi=$ $q \delta x$. The variation of the total intensity arriving at the detectors as a function of this displacement constitutes the signal of the encoder.

To obtain the field distribution just after the second grating, we once again multiply $\varphi_{2 B}$ times the transmittance $t_{2}$. By use of the Fresnel approximation once again, the field propagated to the plane directly before the third grating can be written as

$$
\begin{aligned}
\varphi_{3 B}(x)= & \sum_{n} \sum_{m} a_{n} a_{m} \exp (i \phi m) \exp \{i x[q(n+m) \\
& \left.\left.+q_{0}\right]\right\} \exp \left[-i\left(q n+q_{0}\right)^{2} \frac{z_{1}}{2 k}\right] \exp \{-i[q(n \\
& \left.\left.+m)+q_{0}\right]^{2} \frac{z_{2}}{2 k}\right\} .
\end{aligned}
$$

The intensity distribution associated with this field distribution [Eq. (8)] is

$$
\begin{aligned}
I_{3 B}(x)= & \left|\varphi_{3 B}(x)\right|^{2} \\
= & \sum_{n} \sum_{n^{\prime}} \sum_{m} \sum_{m^{\prime}} a_{n} a_{n^{\prime}} a_{m} a_{m^{\prime}} \exp \left[i \phi\left(m-m^{\prime}\right)\right] \\
& \times \exp \left[i x q\left(n-n^{\prime}+m-m^{\prime}\right)\right] \\
& \times \exp \left[-i q^{2}\left(n^{2}-n^{\prime 2}\right) \frac{z_{1}+z_{2}}{2 k}\right] \\
& \times \exp \left[-i q^{2}\left(m^{2}-m^{\prime 2}\right) \frac{z_{2}}{2 k}\right] \\
& \times \exp \left[-i q^{2}\left(n m-n^{\prime} m^{\prime}\right) \frac{z_{2}}{k}\right] \\
& \times \exp \left[-i q q_{0}\left(n-n^{\prime}\right) \frac{z_{1}+z_{2}}{k}\right] \\
& \times \exp \left[-i q q_{0}\left(m-m^{\prime}\right) \frac{z_{2}}{k}\right] .
\end{aligned}
$$

To simplify expression (9), we make the substitutions $h=n-n^{\prime}$ and $l=m-m^{\prime}$, so we can write

$$
\begin{aligned}
I_{3 B}(x)= & \sum_{l} \sum_{h} \exp (i \phi l) \exp [i x q(h+l)] \\
& \times \exp \left(-i q^{2} h^{2} \frac{z_{1}+z_{2}}{2 k}\right) \exp \left(-i q^{2} l^{2} \frac{z_{2}}{2 k}\right) \\
& \times \exp \left(-i q^{2} l h \frac{z_{2}}{k}\right) \exp \left(-i q q_{0} h \frac{z_{1}+z_{2}}{k}\right) \\
& \times \exp \left(-i q q_{0} l \frac{z_{2}}{k}\right) A(h, l) B(h, l),
\end{aligned}
$$

where

$$
\begin{aligned}
A(h, l)= & \sum_{n^{\prime}} a_{n^{\prime}+h} a_{n^{\prime}} \exp \left(-i q^{2} n^{\prime} h \frac{z_{1}+z_{2}}{k}\right) \\
& \times \exp \left(-i q^{2} n^{\prime} l \frac{z_{2}}{k}\right), \\
B(h, l)= & \sum_{m^{\prime}} a_{m^{\prime}+l} a_{m^{\prime}} \exp \left[-i q^{2} m^{\prime}(l+h) \frac{z_{2}}{k}\right] .
\end{aligned}
$$

Now we must consider the effect of the third grating $G_{3}$ on the intensity distribution given by Eq. (10). The transmittance function for grating $G_{3}$ coincides with that for $G_{1}$. Because the Rhonchi gratings are binary gratings, $\left|t_{i}\right|^{2}=t_{i}$, so $I_{3 A}(x)=I_{3 B}(x)\left|t_{3}(x)\right|^{2}=$ $I_{3 B}(x) t_{3}(x)$. Finally, the intensity distribution directly behind the last grating (where the observation plane is located) is given by

$$
\begin{aligned}
I_{3 A}(x)= & \sum_{n} \sum_{l} \sum_{h} a_{n} \exp (i \phi l) \exp [i x q(h+l+n)] \\
& \times \exp \left(-i q^{2} h^{2} \frac{z_{1}+z_{2}}{2 k}\right) \exp \left(-i q^{2} l^{2} \frac{z_{2}}{2 k}\right) \\
& \times \exp \left(-i q^{2} l h \frac{z_{2}}{k}\right) \exp \left(-i q q_{0} h \frac{z_{1}+z_{2}}{k}\right) \\
& \times \exp \left(-i q q_{0} l \frac{z_{2}}{k}\right) A(h, l) B(h, l) .
\end{aligned}
$$

Now we consider that, in the detection process, the size of the detector is much bigger than $p$, and therefore we detect an intensity distribution averaged over the period of the gratings. This averaged intensity is given by

$$
I(\phi)=\left\langle I_{3 A}(x)\right\rangle=\int_{-p / 2}^{p / 2} I_{3 A}(x) \mathrm{d} x .
$$

If we substitute Eq. (13) into Eq. (14) and make some other substitutions, we obtain

$$
\begin{aligned}
I(\phi)= & \sum_{l} \sum_{h} a_{h+l} \exp (i \phi l) \exp \left(-i q^{2} h^{2} \frac{z_{1}+z_{2}}{2 k}\right) \\
& \times \exp \left(-i q^{2} l^{2} \frac{z_{2}}{2 k}\right) \exp \left(-i q^{2} l h \frac{z_{2}}{k}\right) \\
& \times \exp \left(-i q q_{0} h \frac{z_{1}+z_{2}}{k}\right) \\
& \times \exp \left(-i q q_{0} l \frac{z_{2}}{k}\right) A(h, l) B(h, l) .
\end{aligned}
$$


If we now consider that $z_{1}=z_{2}=z$, as is the case in a reflection optical encoder, expression (15) can be written as

$$
\begin{aligned}
I(\phi)= & \sum_{l} \sum_{h} a_{h+l} \exp (i \phi l) \exp \left(-i q^{2} h^{2} \frac{z}{k}\right) \\
& \times \exp \left(-i q^{2} l^{2} \frac{z}{2 k}\right) \exp \left(-i q^{2} l h \frac{z}{k}\right) \\
& \times \exp \left[-i q q_{0}(2 h+l) \frac{z}{k}\right] A(h, l) B(h, l),
\end{aligned}
$$

which is the main result on which the remainder of this study is based. $I(\phi)$ is a periodic function of $\phi$ that is the signal of the reflection encoder. We study its properties and its dependence on different construction parameters of the encoder in the sections below.

\section{Modulation of the Signal}

Modulation is defined as the difference between the maximum and the minimum signal level of the encoder. One of the most relevant characteristics of a moiré encoder (regardless of whether it operates by reflection) is the dependence of this modulation on the distance between gratings. First, we consider the modulation of the signal when the incident light field is normal to the optical axis, that is, when $q_{0}=$ 0 in expression (16). In this case the total detected intensity is

$$
\begin{aligned}
I(\phi)= & \sum_{l} \sum_{h} a_{h+l} \exp (i \phi l) \exp \left(-i q^{2} h^{2} \frac{z}{k}\right) \\
& \times \exp \left(-i q^{2} l^{2} \frac{z}{2 k}\right) \exp \left(-i q^{2} l h \frac{z}{k}\right) A(h, l) B(h, l) .
\end{aligned}
$$

Because we assume that the incident beam is perfectly collimated and parallel to the optical axis, it seems correct to suppose that the maximum intensity would be obtained when the three gratings were aligned, i.e., when $\phi=0$, and the minimum intensity would be obtained when the second grating was shifted half a period, i.e., when $\phi=\pi$. Then the modulation of expression (17) would be

$$
\begin{aligned}
M_{R}= & I_{\max }-I_{\min } \\
= & \sum_{l \neq 0}\left|a_{l}\right|^{2} \cos \left(\frac{z}{2 k} q^{2} l^{2}\right) \\
& \times\left[\frac{1}{2}+\sum_{n^{\prime}}\left|a_{n^{\prime}}\right|^{2} \exp \left(-i \frac{z}{k} q^{2} n^{\prime} l\right)\right] .
\end{aligned}
$$

Equation (18) must be compared with the expression for the modulation in a transmission encoder,

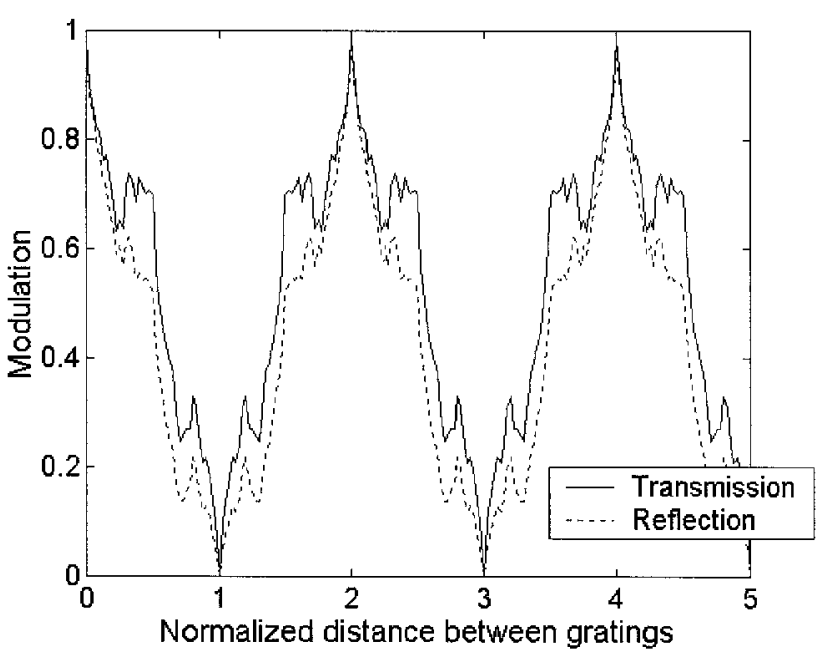

Fig. 3. Variation of the modulation of the signal with the distance $z$ between gratings for the three-grating (reflection) and the twograting (transmission) cases. The distance $z$ is normalized to the Talbot distance.

i.e., a two-grating moiré encoder, that would be given by $^{4}$

$$
M_{D}=\sum_{l \neq 0}\left|a_{l}\right|^{2} \cos \left(\frac{z}{2 k} q^{2} l^{2}\right) .
$$

When the Talbot condition ${ }^{4}$ is met, that is, when $z=n p^{2} / 2 \lambda$, both expressions for the modulation [Eqs. (18) and (19)] have the same value. This means that the locations of the maxima and the minima of the modulation and their values at these extrema are coincident for both expressions. In Fig. 3 a graph of expressions (18) and (19) is shown. The horizontal axis represents the distance $z$ between gratings normalized to the Talbot distance for those gratings. This figure shows that the modulation in the threegrating case is always inferior to that of the twograting scheme. But the difference between both cases is small for the values of $z$ close to the planes of maximum modulation (the difference between both cases is less than $10 \%$ for an interval of $z$ that covers $20 \%$ of a Talbot distance on either side of the location of a Talbot plane). The optical encoder is always configured to work near a Talbot plane because it is the position of maximum modulation, so the lack of modulation, or its steep variation, will not be a significant problem for a reflection encoder compared with a transmission encoder.

\section{Size of the Source}

We want to study the changes that appear in the signal of a reflection encoder when we consider that the source is not a point but that it has a certain size $S$. We consider that the source is centered on the origin of the focal plane and that this center lies on the optical axis of the system.

The total intensity when the incident light field is a planar wave front is given by expression (16). We consider that, when an extended light source is used, 
the light incident upon the system can be treated as an incoherent sum of planar wave fronts with different angles of incidence. The total intensity when we have an extensive light source of size $S$ collimated with a lens of focal length $f$ is given by

$$
I_{S}(\phi)=\int_{-\pi S / f \lambda}^{\pi S / f \lambda} I\left(q_{0}\right) \mathrm{d} q_{0} .
$$

Substituting expression (16) into Eq. (20) and integrating, we get

$$
\begin{aligned}
I_{S}= & \sum_{l} \sum_{h} a_{h+l} \exp (i \phi l) \exp \left(-i q^{2} h^{2} \frac{z}{k}\right) \\
& \times \exp \left(-i q^{2} l^{2} \frac{z}{2 k}\right) \exp \left(-i q^{2} l h \frac{z}{k}\right) A(h, l) B(h, l) \\
& \times \operatorname{sinc}\left[\pi \frac{S}{f} \frac{z}{p}(2 h+l)\right],
\end{aligned}
$$

where $\operatorname{sinc}(x)=\sin (x) / x$. The modulation of the signal of the encoder is given by

$$
\begin{aligned}
M_{S T}= & I_{S \max }-I_{S \min } \\
= & \sum_{l \neq 0}\left|a_{l}\right|^{2} \cos \left(\frac{z}{2 k} q^{2} l^{2}\right) \operatorname{sinc}\left(\pi \frac{S}{f} \frac{z}{p} l\right) \\
& \times\left[\frac{1}{2}+\sum_{n^{\prime}}\left|a_{n^{\prime}}\right|^{2} \exp \left(-i \frac{z}{k} q^{2} n^{\prime} l\right)\right] .
\end{aligned}
$$

In an encoder that works as a two-grating system the modulation when an extended source is used is given by $^{3}$

$$
M_{S D}=\sum_{l \neq 0}\left|a_{l}\right|^{2} \cos \left(\frac{z}{2 k} q^{2} l^{2}\right) \operatorname{sinc}\left(\pi \frac{S}{f} \frac{z}{p} l\right) .
$$

Comparing Eqs. (22) and (23) with expressions (18) and (19), we can see that the effect of the extended size of the source is equivalent in the three-grating and the two-grating systems. In both cases there is a loss of modulation proportional, in a first approximation, to $\operatorname{sinc}(\pi S z / p f)$.

\section{Alignment of the Source}

We consider now the problems that can arise from the misalignment of the light source with respect to the optical axis of the system. Under a geometrical approximation it is clear that the inclination of the incident wave front will have a strong influence on the signal of the encoder. In Fig. 4, we can see that, if the incident wave front is tilted by an angle of $\theta=$ $p / 4 z$, the shadow of the first grating cast onto the plane of the third grating is in counterphase with this last grating. In this case, we would have zero intensity on the observation plane for any position of the second grating; the encoder would not give any signal at all.

We can study the effects of the misalignment of the source more rigorously by using the result obtained in expression (16). The light source is considered to

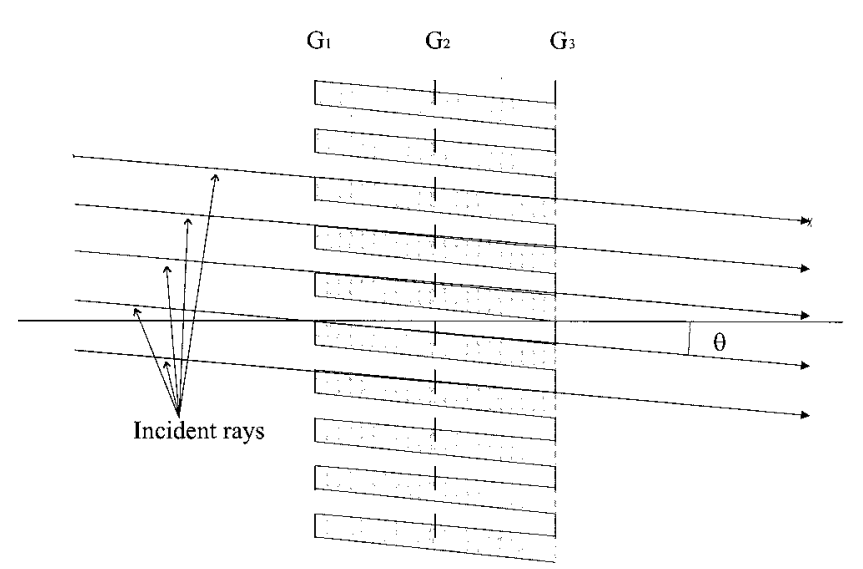

Fig. 4. Under a geometric approximation, when the shadow of the first grating for a given angle of incidence is projected onto the third grating it completely darkens the observation plane.

be point, as in the following calculations. We also consider, for simplicity, that the distance between the gratings meets the Talbot plane condition, $z=$ $n p^{2} / 2 \lambda(n \in N)$. In this case the intensity on the observation plane is given by

$$
\begin{aligned}
I_{T}(\phi)= & \frac{1}{8}+\sum_{h \neq 0} a_{h}{ }^{2}\left[\cos (\phi h) \cos \left(q q_{0} h \frac{z}{k}\right)\right. \\
& \left.+\frac{1}{2} \cos \left(2 q q_{0} h \frac{z}{k}\right)\right] .
\end{aligned}
$$

The corresponding modulation is given by

$$
M_{T}=2 \sum_{h \neq 0} a_{h}^{2} \cos \left(q q_{0} h \frac{z}{k}\right) .
$$

In Eq. (25) the modulation varies periodically with the tilt of the incident light field; it reaches its maximum for the values of $q_{0}$ such that $q_{0}=n \pi p / \lambda z$, and it reaches 0 for $q_{0}=(2 n+1) \pi p / 2 \lambda z$. Not only the modulation of the signal varies with $q_{0}$ but also its shape. In Fig. 5, we show how the signal varies from a triangular shape for $q_{0}=0$ to a flat line for $q_{0}=$ $\pi p / 2 \lambda z$, passing through different trapezoidal curves for intermediate values of $q_{0}$.

In a reflection encoder in which the system is, in fact, working as a three-grating system, there is a special requirement on the alignment of the source that does not exist in a transmission encoder working as a two-grating system. Because $q_{0}=2 \pi \theta / \lambda$, we can see from expression (25) that, if $\theta$ is larger than $p / 8 z$, the modulation of the signal of the encoder will be down by almost a factor of 2.0. If we want to build an encoder with a well-modulated signal, taking into account that $\theta=D / f$, we would have a condition for the alignment of the source that would be expressed as

$$
\frac{D}{f}<\frac{p}{8 z}
$$




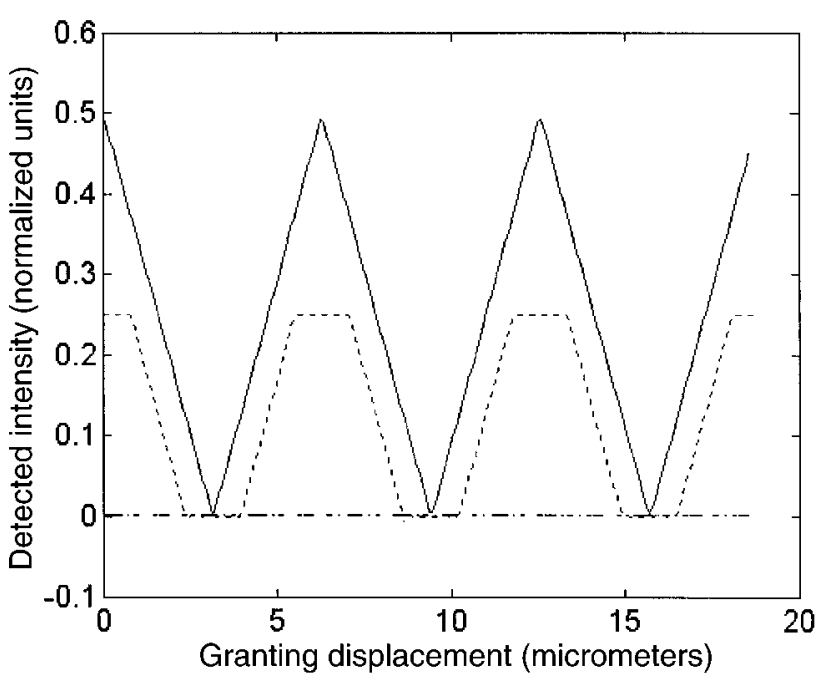

Fig. 5. Variation of the signal given by the encoder plotted as a function of the inclination of the incident beam. The solid curve represents $q_{0} \lambda z / p=0$; the dashed curve represents $q_{0} \lambda z / p=\pi / 4$; the dashed-dotted line represents $q_{0} \lambda z / p=\pi / 4$.

Condition (26) imposes a limit on the manufacturing tolerances of the system that must be taken into account when designing a commercial optical encoder.

\section{Defocusing}

As we show below, when the light source is positioned out of the lens's focal plane different degrees of signal phase and signal modulation can occur at different zones of the reading grating. In a case in which we had a single monolithic detector covering all the area of the reading grating the lack of signal uniformity across the grating should be averaged. Nevertheless, the reading grating is usually divided into two or more zones (observation windows), each with its own detector. A scheme of an arrangement with different reading windows is shown in Fig. 6. The reason for this configuration is that encoders are designed to provide two or more signals with phase differences between them. ${ }^{1}$ Those shifted signals allow the de-

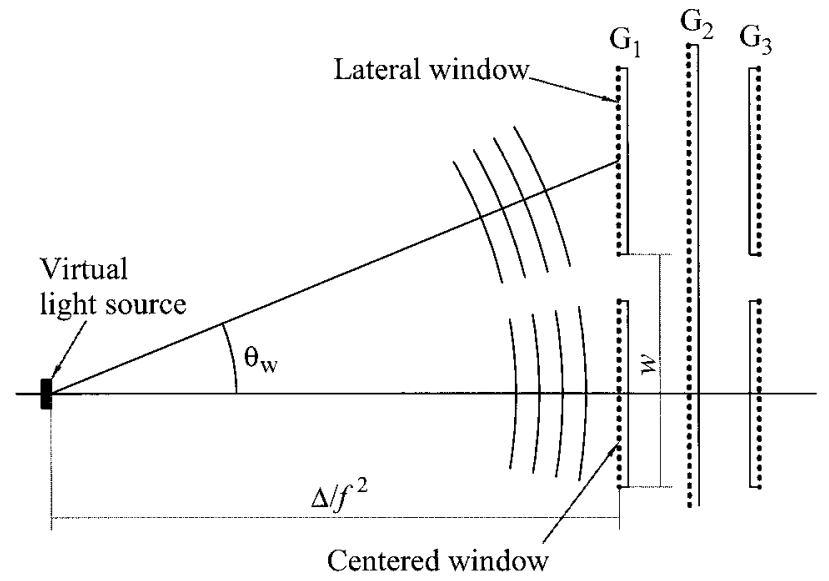

Fig. 6. Schematic representation of the incident field on each observation window when the light source is defocused. termination of the direction of movement. Shifted signals also provide the encoder with the possibility of interpolating the digital count, resulting in an electronic increase of the encoder resolution.

Each signal is generated by the photodetector located behind each observation window. The relative positions of the windows depend on the encoder design as well as on the desired phase shift. With this kind of arrangement the lack of uniformity of the phase and the modulation of the overall signal turns into relative variations of the phase and the amplitude of the signals obtained at each window. This variation negatively affects the analog and the digital algorithms that compute interpolation and movement direction, eventually producing malfunctioning of the counter.

To evaluate the effect of defocusing on the aforementioned error, we consider the most usual configuration: two windows with a mutual phase shift of $\pi / 2$. One of these windows is centered on the optical axis of the system, and the other one is displaced along the $x$ axis by a distance $w$. Both observation windows have a phase difference of $\pi / 2$ between them, so the distance $w$ must be equal to $N p+p / 4$, where $N$ is an integer large enough so that $N p$ is larger than the size of the windows.

If the source is shifted along the optical axis a small distance $\Delta$ out of the focal plane the incident light field is a spherical wave front with a radius of curvature $R$ given by

$$
1 / R \approx \Delta / f^{2}
$$

where the distance between the lens and the first grating is neglected with respect to the radius of curvature $R$, which for small deviations from the focal plane will have large values. According to expression (27), the wave front can be approximated locally by a planar wave front. On the observation window that is centered on the optical axis the incident light field can be approximated by a planar wave front that is normal to the optical axis. On the observation window that is shifted laterally by a distance $w$ the incident light field can be approximated by a planar wave front that is tilted by an angle of

$$
\theta_{w}=w / R \approx w \Delta / f^{2}
$$

With these considerations [expressions (27) and (28)] the total intensity on each window is given by

$$
\begin{aligned}
I_{O}= & \sum_{l} \sum_{h} a_{h+l} \exp (i \phi l) \exp \left(-i q^{2} h^{2} \frac{z}{k}\right) \\
& \times \exp \left(-i q^{2} l^{2} \frac{z}{2 k}\right) \exp \left(-i q^{2} l h \frac{z}{k}\right) A(h, l) B(h, l)
\end{aligned}
$$


for the centered window and

$$
\begin{aligned}
I_{w}= & \sum_{l} \sum_{h} a_{h+l} \exp \left[i\left(\phi+\frac{\pi}{2}\right) l\right] \\
& \times \exp \left(-i q^{2} h^{2} \frac{z}{k}\right) \exp \left(-i q^{2} l^{2} \frac{z}{2 k}\right) \\
& \times \exp \left(-i q^{2} l h \frac{z}{k}\right) \exp \left[-i q \theta_{w}(2 h\right. \\
& +l) z] A(h, l) B(h, l) .
\end{aligned}
$$

For simplicity, we consider that the separation $z$ between gratings meets the Talbot condition, so expressions (29) and (30) can be written as

$$
\begin{aligned}
I_{O}(\phi)= & \frac{1}{4}+\sum_{h \neq 0}\left|a_{h}\right|^{2} \cos (\phi h), \\
I_{W}(\phi)= & \frac{1}{8}+\sum_{h \neq 0}\left|a_{h}\right|^{2}\left\{\cos \left[\left(\phi+\frac{\pi}{2}\right) h\right] \cos \left(h q \theta_{w} z\right)\right. \\
& \left.+\frac{1}{2} \cos \left(2 h q \theta_{w} z\right)\right\},
\end{aligned}
$$

respectively. There is a loss of modulation in the lateral window that is given by Eq. (25). But, on the other hand, the phase difference between both signals remains constant at $\pi / 2$ when the light source is defocused. This situation is not true in the case of a transmission encoder that works as a two-grating system; in this case, the modulation would remain constant, but the phase shift between different windows would vary. The stability of the phase difference between different observation windows would be an advantage of using a three-grating configuration.

\section{Experimental Results}

We now show some experimental results that were obtained with a reflection encoder like the one described in the previous sections. The experimental setup was devised to test the analytical results presented above, and it is described in Fig. 1. The system has a reflection grating and a transmission grating with two observation windows placed on it; both gratings have a period of $100 \mu \mathrm{m}$. As was mentioned above, the transmission grating acts as both the first and the third grating in a three-grating moiré system. This experimental system is equivalent to the system that we studied in Sections 1-5 if we consider that the inclination of the incident beam has no relevance for the analytical study because the gratings are aligned along the $x$ axis.

The two observation windows have a mutual phase difference of $\pi / 2$, and they are separated by a distance of $5.4 \mathrm{~mm}$. As collimating lenses, we used plane-aspheric singlets with back focal lengths of 11 and $18 \mathrm{~mm}$. We used quasi-monochromatic sources [infrared-emitting diodes (IRED's) and laser diodes] that emit at $780 \mathrm{~nm}$. For this wavelength and a grating period of $100 \mu \mathrm{m}$ the first Talbot plane is

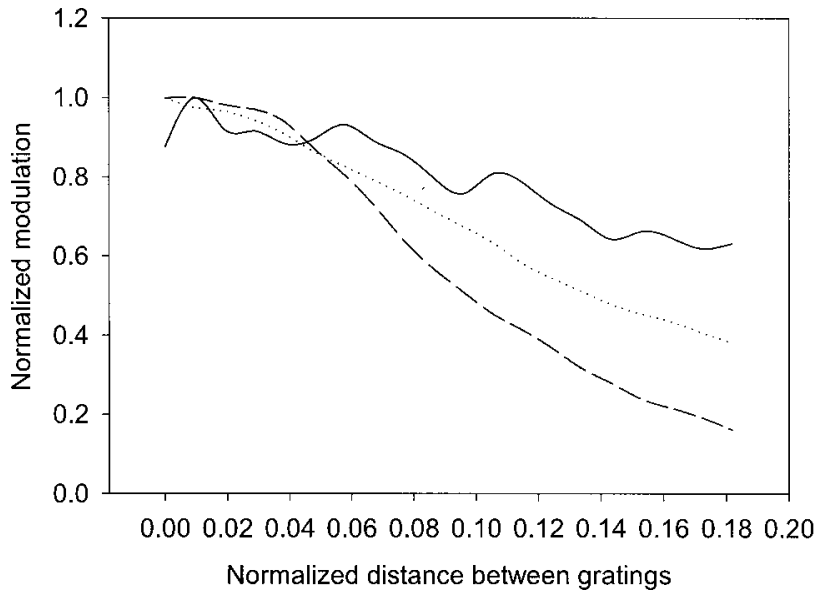

Fig. 7. Modulation curves for different sizes of the light source. The solid curve represents the results from the diode laser; the dotted curve represents the results from the $200-\mu \mathrm{m}$ emitting IRED laser; the long-dashed curve represents the results from the $350-\mu \mathrm{m}$ emitting IRED laser.

obtained for a separation between gratings of 12.8 mm.

The experimental setup that we used allows for control of the position of the light source with respect to the lens with a precision of $\pm 1.0 \mu \mathrm{m}$. Also, the reflection grating is attached to a set of two stepdriven motors, so we can control its displacement along the $x$ and the $z$ axes with a resolution of \pm 0.25 $\mu \mathrm{m}$ and a precision of $\pm 1 \mu \mathrm{m} / 10 \mathrm{~cm}$.

The magnitude that is to be measured is the modulation of the signal of the encoder, which is understood to be the peak-to-peak value of the signal. The results are presented as graphs that show the variation of this modulation as a function of $z$ for different configurations of the encoder.

First, we took some measurements with three different sources, taking care that they were placed on the back focal plane of the lens and that the incident light beam was normal to the optical axis of the system. The first source used was a transversal monomode laser diode emitting at $780 \mathrm{~nm}$. Because of the spatial coherence of the emitted field, this source can be considered to be punctual, with the collimation being limited by the lens performance and, ultimately, by diffraction. The other two sources were two IRED's also emitting in a band centered about $780 \mathrm{~nm}$. One of the IRED's had a dome-shaped emitting surface with a diameter of $200 \mu \mathrm{m}$; the other one had a square emitting surface with a side length of $350 \mu \mathrm{m}$. These measurements were made by use of a collimating lens with a focal length of $11.0 \mathrm{~mm}$.

The results obtained with these three different sources are shown in Fig. 7. It can clearly be seen how the slope of the modulation curve becomes steeper as the size of the source increases; the result is consistent with expression (22).

The next set of measurements was made to verify the influence of misalignment of the source on the performance of the encoder. These measurements 


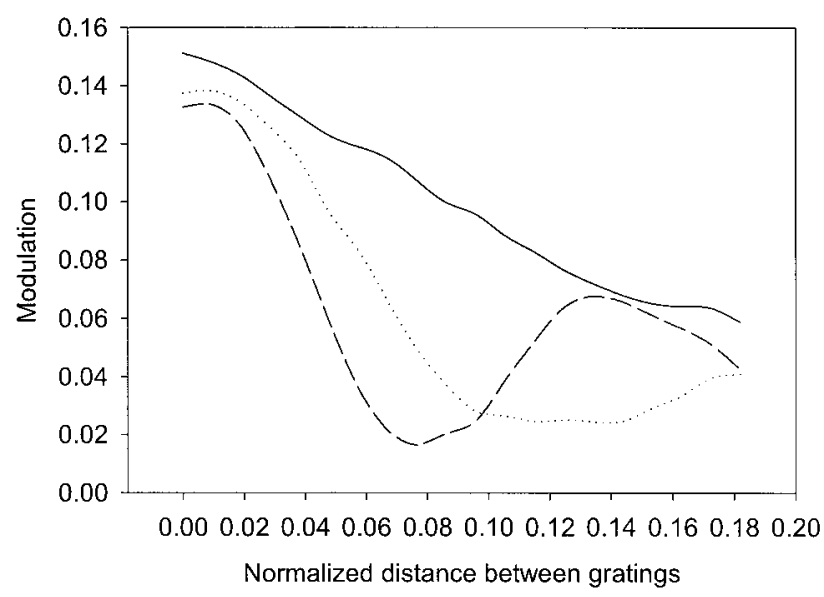

Fig. 8. Modulation curves for different angles $\theta$ of the incident beam. The solid curve represents normal incidence; the dotted curve represents an angle of incidence of $\theta=2^{\circ}$; the long-dashed curve represents an angle of incidence of $\theta=4^{\circ}$.

were made by use of the IRED with the domeshaped emitting surface and a lens with a focal length of $18 \mathrm{~mm}$. The results of these measurements are shown in Fig. 8. Each curve corresponds to a different angle of the incident light beam with respect to the $x$ axis of the system. Each of these curves could be regarded as the same curve that corresponds to the case of normal incidence but that is affected by a sinusoidal envelope that periodically decreases as the angle of incidence increases. This is the behavior predicted by expression (25). Although expression (25) was obtained for only values of $z$ that satisfied the Talbot condition, we can assume that the sinusoidal influence on the modulation that is due to the tilt of the wave front is true for any value of $z$. This assumption seems to be corroborated by the experimental results shown in Fig. 8. Expression (25) was regarded as a function of $q_{0}$ (the tilt of the incident wave front) for a constant value of $z$; it can be regarded now as a function of $z$ for a constant value of $q_{0}$. This is what each curve in Fig. 8 represents.

Another set of measurements was made to verify the variations of the signals from different observation windows when the light source is defocused. In Fig. 9(a), we show the curves that were obtained for the central observation window for different values of the defocusing parameter $\Delta$. In Fig. 9(b), we show the same results for the lateral window. It can be seen how a sinusoidal envelope superimposed on the curves (similar to the one observed in Fig. 8) appears in the lateral window as we increase the value of $\Delta$; that behavior is not observed in the central window. The behavior observed in Figs. 9(a) and 9(b) is consistent with expressions (31) and (32), respectively. The similarity between Fig. 9(b) and Fig. 8 seems to corroborate the supposition that was made in the Section 5 that stated that the effect of defocusing on a lateral window should be similar to the effect of tilting the incident wave front.

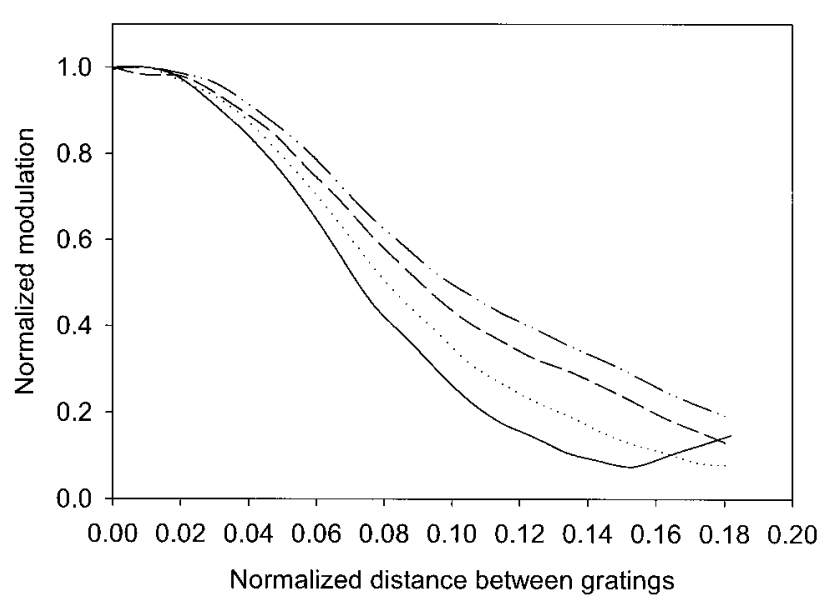

(a)

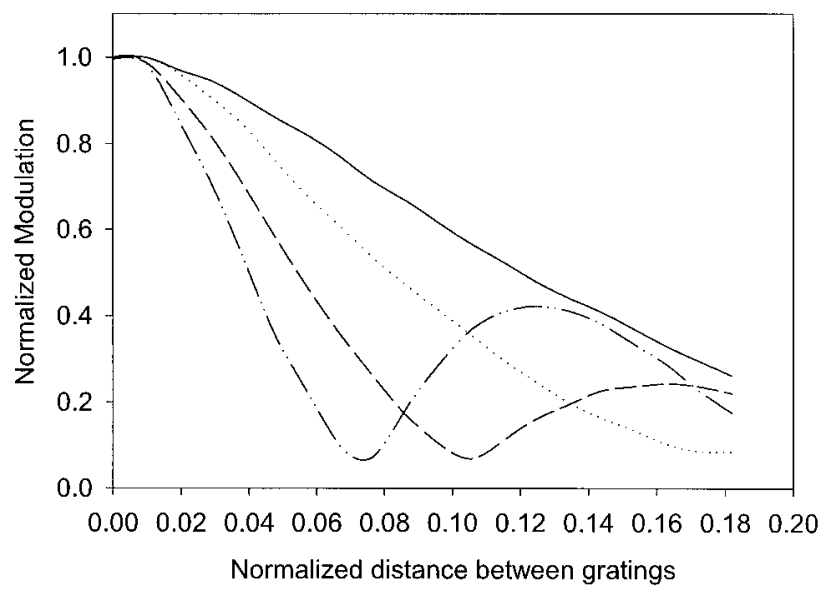

(b)

Fig. 9. Modulation curves for different defocusing positions for (a) the central observation window and (b) the lateral observation window. The solid curve represents the results for no defocus, $\Delta=0$; the dotted curve represents results for a defocus value of $\Delta=$ $1.0 \mathrm{~mm}$; the long-dashed curve represents results for a defocus value of $\Delta=2.0 \mathrm{~mm}$; the dashed-dotted curve represents results for a defocus value of $\Delta=2.5 \mathrm{~mm}$.

\section{Conclusions}

In this paper, we have studied the implications of considering a reflection optical encoder as a threegrating moiré system. We have obtained a general equation [Eq. (15)] that allows the calculation of the observed intensity as a function of the spatial frequency on the first grating when a shifted punctual source is used. This equation can be integrated to take into account the effect of finite size sources, source misalignment, and defocusing. Simple examples for all these situations have been analyzed, and the corresponding equations [Eqs. (21), (24), and (30)] for the observed intensity have been presented. We have shown that the alignment and the defocusing of the light source have a strong influence on the signal of the encoder. This behavior does not appear when the encoder is studied as a two-grating moiré system. Experimental results have been presented 
that are in good qualitative agreement with the theoretical results.

This research was supported by a project from the Comisión Interministerial de Ciencia y Tecnología (CICYT) under grant TAP 98-0862.

\section{References}

1. K. Patorski, Handbook of the Moiré Fringe Technique (Elsevier, Amsterdam, The Netherlands, 1993), Chap. 5, pp. 99-139.

2. F. Talbot, "Facts relating to optical science: Number IV," Philos. Mag. 9, 401-407 (1836).

3. A. Olszak and L. Wronkowski, "Analysis of the Fresnel field of a double diffraction system in the case of two amplitude diffraction gratings under partially coherent illumination," Opt. Eng. 36, 2149-2157 (1997).

4. E. Keren and O. Kafri, "Diffraction effects in moiré deflectometry,” J. Opt. Soc. Am. A 2, 111-120 (1985).

5. K. Patorski, Moiré Metrology (Pergamon, New York, 1998).

6. G. N. Rassudova, "Moiré interference fringes in a system con- sisting of a transmission and a reflection diffraction grating. Part I," Opt. Spectrosc. 22, 73-78 (1967); G. N. Rassudova, "Moiré interference fringes in a system consisting of a transmission and a reflection diffraction grating. Part II," Opt. Spectrosc. 22, 255-258 (1967); G. N. Rassudova, "Moiré interference fringes in a system consisting of a transmission and a reflection diffraction grating. Part III," Opt. Spectrosc. 22, 335-340 (1967).

7. L. Liu, X. Liu, and L. Ye, "Joint Talbot effect and logic-operated moiré patterns,” J. Opt. Soc. Am. A 7, 970-976 (1990).

8. L. Wronkowski, "Diffraction model of an optoelectronic displacement measuring transducer," Opt. Laser Technol. 27, 81-88 (1995).

9. L. Wronkowski, "Opto-electronic analog-impulse transducer accuracy from the point of view of diffraction phenomena," in New Measurement Technology to Serve Mankind: Acta IMEKO 1985, Volume III. Measurement in Mechanics and Laser Metrology, G. Striker, T. Boromisza, and T. Kemeny, eds. (OmikkTechnoinform, Budapest, Hungary, 1985), pp. 445-462. 